\title{
Initial Clinical Outcomes of Minimally Invasive Lateral Lumbar Interbody Fusion in Degenerative Lumbar Disease: A Preliminary Report on the Experience of a Single Institution with 30 Cases
}

\author{
Young Cheol Na, Hyo Sang Lee, Dong Ah Shin, Yoon Ha, \\ Keung Nyun Kim, Do Heum Yoon \\ Department of Neurosurgery, Yonsei University College of Medicine, Severance Hospital, The Spine \\ and Spinal Cord Institute, Seoul, Korea
}

\begin{abstract}
Objective: The object of this study was to evaluate the clinical and radiological outcomes of minimally invasive lateral lumbar interbody fusion.

Methods: This study included 30 patients who underwent minimally invasive lateral lumbar interbody fusion at our hospital between May 2011 and February 2012 for the following diagnoses: degenerative disc disease, adjacent-segment degeneration, degenerative spondylolisthesis and lumbar degenerative scoliosis. Pain assessment was reported from 0 to 10 using a subjective visual analog scale (VAS) upon admission and at every follow-up day. Lumbar X-rays were obtained in the standing position upon admission and the 1st and 5th postoperative day, and at every follow-up day after the operation. The heights of the intervertebral disc space and neural foramen were measured using an electronic caliper with the PACS software. The surgical outcome was assessed as excellent, good, fair or poor using the Odom scale at the last follow-up. Results: The mean VAS for low back pain were $4.93 \pm 1.47$ on admission and $2.01 \pm 1.35$ at last follow-up, respectively, and for leg pain, the scores were $4.87 \pm 2.16$ on admission and $1.58 \pm 1.52$ at last follow-up. The mean height of intervertebral disc space increased by $34 \%(7.93 \pm 2.33$ preoperatively, and $11.09 \pm 4.33$ immediately after surgery, $p<0.01)$. The mean height of neural foramen also increased by $6.4 \%$ without any statistical significance $(19.17 \pm 2.84$ preoperatively, and $20.49 \pm 4.50$ immediately after the surgery). Minimally invasive lateral lumbar interbody fusion was successful in 27 patients (90\%) at last follow-up. Surgical complications were reported as transient postoperative thigh sensory changes (5 patients, $16.7 \%)$, transient psoas muscle weakness ( 3 patients, 10\%), cage migration (2 patients, 6.7\%), lumbar plexus injury (1 patient, $3.3 \%$ ), and pain aggravation (1 patient, 3.3\%).

Conclusion: The minimally invasive lateral lumbar interbody fusion is a safe and effective procedure for treating degenerative lumbar disease with good outcomes and moderate complications. Further follow-up is necessary to establish its safety and efficacy.
\end{abstract}

Key Words: Spinal fusion $\cdot$ Treatment outcome $\cdot$ Complication

\section{INTRODUCTION}

After the first laparoscopic lumbar discectomy was performed and described by Obenchain in 1991 ${ }^{24)}$, minimally invasive spine surgery (MISS) has been an attractive treatment

- Received: April 13, 2012 - Revised: September 4, 2012

- Accepted: September 26, 2012

Corresponding Author: Keung Nyun Kim, MD

Department of Neurosurgery, Yonsei University College of Medicine

134 Shinchon, Seodaemoon-Gu, Seoul 120-752, Korea

TEL: +82-2-2228-2152, FAX: +82-2-393-9979

E-mail: knkim@yuhs.ac modality for surgeons. The advantages of MISS include less tissue damage, less estimated blood loss, less postoperative pain, shorter hospital stays, and a faster return to daily living activities ${ }^{3,6,12,16,23)}$. The most recently introduced MISS technique is the lateral transpsoas approach for lumbar interbody fusion. This minimally invasive lateral lumbar interbody fusion (MIS LLIF), also known as direct lumbar interbody fusion (DLIF) or extreme lumbar interbody fusion (XLIF), has been used in the surgical treatment of degenerative lumbar disease in adults, including degenerative scoliosis ${ }^{2,311)}$. These degenerative lumbar diseases often require stabilization procedures ${ }^{11}$. While lumbar stabilization was traditionally obtained through the posterior approach with open procedures, the technical 
advantages that support the anterior column have gained the interest of surgeons due to its improved stability because of the large surface area ${ }^{22}$. However, the associated morbidity with open anterior procedures including vascular injury, injury to the retroperitoneal structures, injury to the ilioinguinal or iliohypogastric nerves, and retrograde ejaculation ${ }^{8,18,21)}$ hasmotivated surgeons to seek alternative modalities for minimally invasive techniques like MIS LLIF. Moreover, the recently reported benefit of the correction of coronal balance has expanded the use of MIS LLIF ${ }^{1,22}$. While a number of authors have reported on the surgical techniques of MIS LLIF, report on the surgical outcomes is not common. The object of this study was to evaluate the clinical and radiological outcomes of MIS LLIF.

\section{MATERIALS AND METHODS}

\section{Patient demographics}

This study involved 30 patients who underwent MIS LLIF at our hospital between May 2011 and February 2012. Five men and 25 women participated in this study. The age of the patients at the time of the surgery ranged from 50-79 years (mean, 62 years). All patients were followed clinically and radiologically for at least 6 months. The following factors were evaluated: back and leg pain, the heights of the intervertebral disc space and neural foramen, surgical outcome, and surgical complications. Pain intensity was reported from 0-10 using a subjective visual analog scale (VAS; $0=$ no pain; $10=$ the worst pain imaginable). The heights of the intervertebral disc space and neural foramen were measured in the lumbar X-rays. Surgical outcome was assessed as excellent, good, fair or poor using the Odom scale at last follow-up. All patients had a history of radiculopathy refractory to conservative treatment for a minimum of 6 weeks. The preoperative diagnoses were lumbar spondylosis in 7 patients, degenerative scoliosis in 9 patients, adjacent-segment degeneration in 8 patients, and spondylolisthesis in 6 patients (Table 1).

\section{Operative data}

The surgical technique was basically same as Ozgur described previously ${ }^{15}$. The patients were placed in a true $90^{\circ}$ lateral decubitus position on a radiolucent table. The table was flexed at the interspace of interest to increase the distance between the iliac crest and the rib cage, especially useful at the upper lumbar levels and at L4-L5. The anatomic locations of the ribs and of the iliac crest were carefully reviewed so as not to limit the exposure from L1-2 to L4-5. In this study, the L5-S1 disc level was excluded because that superior edge of the iliac crest limits the potential exposure of L5-S1 disc level. Using a K-wire, the center of the disc of interest was localized on the lateral radiograph. A corresponding location was marked on the skin for the incision. After the skin incision, gentle retroperitoneal transpsoas dissection was performed. The peritoneum was swept away anteriorly. Care was taken to minimize trauma to the psoas muscle, which was parted between the middle and anterior third ensuring that the nerves of the lumbar plexus were located posteriorly and outside the operative corridor. A tubular retractor was inserted and docked on the lateral border of the psoas directly over the intervertebral disc space confirmed by AP and lateral fluoroscopy. As the dilator was advanced through the psoas muscle, a continuous search was made for the stimulus threshold that elicited an EMG response on the bilateral vastus medialis, tibialis anterior, biceps femoris, and medial gastrocnemius myotomes. A discectomy and interbody implant placement was performed under direct vision. A thorough discectomy and endplate preparation were performed to increase the interbody fusion rate. The bony endplate was kept to reduce the risk of graft subsidence. The incision was closed with the standard technique. The cages were packed with $\beta$-tricalcium phosphate (ChronOS $^{\mathrm{TM}}$ strip, Synthes ${ }^{\circledR}$ Spine, Inc., PA, USA) and demineralized bone matrix (DBX ${ }^{\circledR}$ putty, Dentsply, Inc. CL, USA). Graft lengths ranged from 45 to $55 \mathrm{~mm}$, with heights from 8 to $12 \mathrm{~mm}$ and had a total lordosis of $6^{\circ}$. Posterior augmentation was performed in 14 cases (47\%) and the cage alone was done in 16 cases (53\%). The methods for posterior augmentation included conventional pedicle screws, percutaneous screws (CD Horizon $^{\circledR}$ Sextant $^{\mathrm{TM}}$, Medtronic Sofamor Danek, Inc., MN, USA) and interspinous fixation (Coflex- $\mathrm{F}^{\circledR}$, Paradigm spine, Inc., NY, USA).

Table 1. Patient demographics

\begin{tabular}{lc}
\hline \hline Variable & Number of patients \\
\hline Male/Female & $5: 25$ \\
Mean age in yrs (range) & $62(50-79)$ \\
Diagnosis & 30 \\
Lumbar spondylosis & 7 \\
Degenerative scoliosis & 9 \\
Adjacent-segment degeneration & 8 \\
Spondylolisthesis & 6 \\
DLIF Level & 45 \\
L1-2 & 6 \\
L2-3 & 11 \\
L3-4 & 15 \\
L4-5 & 13 \\
\hline
\end{tabular}




\section{Radiographic analysis}

Anteroposterior and lateral X-rays of the lumbar spine were obtained upon admission and the $1^{\text {st }}$ and $5^{\text {th }}$ postoperative day and at every follow-up day after the operation. Lumbar computed tomography (CT) was obtained at the $6^{\text {th }}$ month and $1^{\text {st }}$ year after the operation. The heights of intervertebral disc space and neural foramen were measured upon admission and the 1st day after the operation, using an electronic caliper with the PACS software. In addition, endplate damage and settling of the cages were carefully reviewed. Endplate damage was classified into 4 subtypes: Grade 0 represented a normal endplate without damage; Grade I represented a breach of the endplate at one side (anterior or posterior) of the cage; Grade II represented damage to the endplate at both the anterior and posterior sides of the cage; and Grade III signified endplate damage with cage subsidence of more than one-third of the cage height in the vertebral body ${ }^{22)}$.

\section{Data analysis}

The results are expressed as the means \pm standard deviation. Student's t-test was used to assess the statistical differences of the VAS scores and radiologic outcomes at each time point between the groups using the SAS software for Windows (SAS Institute Inc., Cary, NC, USA). A p-value $<0.05$ was considered significant.

\section{RESULTS}

\section{Operative data}

A total of 45 levels were treated with MIS LIF in 30 patients. Nineteen patients were treated at as single level and 11 patients were treated at multiple levels. Among the multilevel patients, 4 patients had 3 levels treated and 7 patients had 2 levels treated. MIS LLIF was performed across an average of 1.48 levels per patient from L1-2 to L4-5 (Table 1). The average operative time was $167 \pm 88$ minutes and the average blood loss was $268 \pm 366 \mathrm{ml}$, including the posterior augmentation cases. In the multilevel cases, a single skin incision was made in 5 cases, a double skin incision in 5 cases, and a triple skin incision in 1 case. Standalone cage was performed in 16 cases (53\%). The remaining 14 were all posterior augmented cases (47\%). Among the posterior augmented cases, 8 cases were instrumented with a conventional pedicle screw system, 4 cases with a percutaneous screw system (CD Horizon ${ }^{\circledR}$ Sextant ${ }^{\mathrm{TM}}$, Medtronic Sofamor Danek, Inc., MN, USA), and 2 levels with an interspinous fixation system (Coflex- $\mathrm{F}^{\circledR}$, Paradigm spine, Inc., NY, USA).

\section{Radiographic outcome}

The mean height of intervertebral disc space was 7.93 \pm $2.33 \mathrm{~mm}$ preoperatively and $11.49 \pm 4.33 \mathrm{~mm}$ postoperatively, with an increase of $34 \%(p<0.001$, Fig. 1). The mean height of neural foramen was $19.17 \pm 2.84 \mathrm{~mm}$ preoperatively and $20.49 \pm 4.50 \mathrm{~mm}$ postoperatively, with an increase of $6.4 \%$ with no statistical significance. Grade 1 endplate damage was observed in 11 levels (24\%). Among them, 7 levels were in the standalone cage group and 4 levels in the posterior augmentation group. Cage settling was observed in 33 levels (62 \%) (Fig. 2). In the standalone cage group, cage settling was observed in 20 levels (87\%): 7 levels with endplate damage and

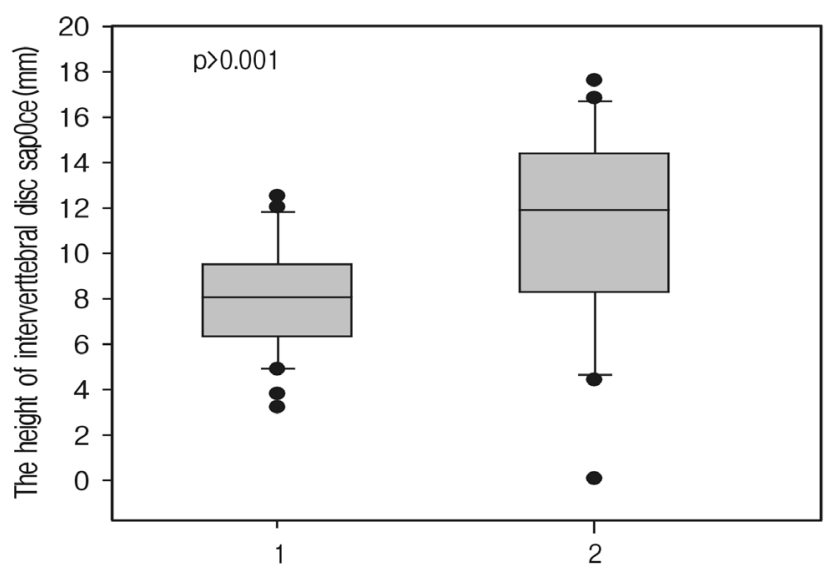

Fig. 1. Bar graph showing changes in the height of the intervertebral disc space (1: preoperatively, 2: at last follow-up).
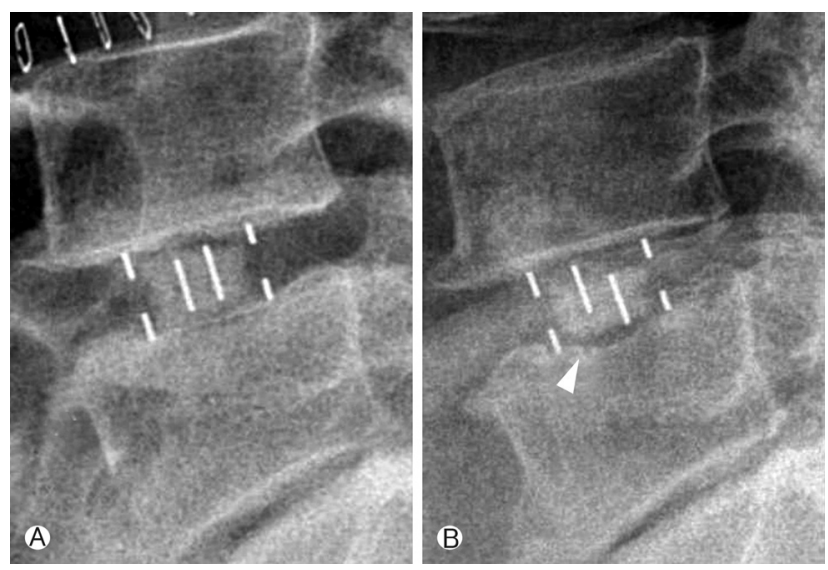

Fig. 2. Cage settling case. (A) immediate postoperative lateral plain radiograph showing an intact endplate after standalone cage surgery. (B) 1 month postoperative lateral plain radiograph showing cage settling (white arrow) after standalone cage surgery. 
Table 2. Cage settling with or without endplate damage for each procedure

\begin{tabular}{lccl}
\hline \hline & $\begin{array}{c}\text { With end plate } \\
\text { damage }\end{array}$ & $\begin{array}{c}\text { Without end } \\
\text { plate damage }\end{array}$ & RR \\
\hline Cage stand alone & $7 / 7(100 \%)$ & $13 / 16(81 \%)$ & 1.23 \\
Posterior augmentation & $4 / 4(100 \%)$ & $9 / 18(50 \%)$ & 2 \\
$\begin{array}{l}\text { Posterior augmentation } \\
\text { (except scoliosis) }\end{array}$ & $4 / 4(100 \%)$ & $4 / 7(57 \%)$ & 1.75 \\
\hline
\end{tabular}

13 levels without endplate damage $(\mathrm{R}=1.23)$. In the posterior augmented group, cage settling was found in 13 levels (59\%): 4 levels with endplate damage and 9 levels without endplate damage $(R R=2)$. Except for 11 degenerative scoliosis levels in the posterior augmentation cases, cage settling was found in 4 levels with endplate damage and 4 levels without endplate damage $(R R=1.75$, Table 2). All of the 11 levels with endplate damages, whether with a standalone cage or posterior augmentation, progressed to cage settling.

\section{Clinical outcome}

Follow-up was accomplished in all patients for at least 6 months. The mean VAS scores for low back pain were 4.93 \pm 1.47 upon admission, and $2.01 \pm 1.35$ at last follow-up. For leg pain, the mean VAS scores was $4.87 \pm 2.16$ upon admission, and $1.58 \pm 1.52$ at last follow-up $(\mathrm{p}<0.001)$. MIS LLIF was successful in 27 patients at last follow-up (90\%): excellent in 12 patients (40\%), good in 15 patients (50\%), fair in 2 patients (6.7\%), and poor in 1 patient (3.3\%).

\section{Complications}

There were 14 complications (46\%) (Table 3). There were cage migrations in 2 cases, genitofemoral numbness in 5 cases, psoas weakness in 3 cases, infection in 1 case, pain aggravation in 1 case, and lumbar plexus injury in 1 case. Genitofemoral numbness was transient in all cases and improved after a few days. Psoas weakness was also transient in all cases and improved after a few months of follow-up. The case with postoperative infection improved with antibiotics. Leg weakness by lumbar plexus injury persisted for 6 months after the operation with a motor grade of 2 at the last follow-up, although minimal improvement was achieved through rehabilitation.

In this study, 3 patients underwent a second operation for revision. Two patients underwent a second operation because of cage migration. One of them required a third operation because of vertebral body fractures. The other reason for revision was pain aggravation in 1 patient. In the pain aggravation patient, MIS LLIF was performed at the L4-5 level. The
Table 3. Detailed list of complications

\begin{tabular}{lcl}
\hline \hline Complication & $\begin{array}{c}\text { Number } \\
\text { of Patients }\end{array}$ & Comments \\
\hline Neurologic complications & 9 & \\
Thigh sensory change & 5 & Transient (Few days) \\
Psoas weakness & 3 & Transient (Few months) \\
Lumbar plexus injury & 1 & Still (POD 6 months) \\
Infection & 1 & Antibiotics \\
Cage related & 2 & \\
$\quad$ Cage migration & 2 & Posterior stabilization \\
Pain aggravation & 1 & Posterior decompression \\
\hline
\end{tabular}

preoperative leg pain was relieved immediately after the first operation, but the patient suffered from Lt L5 dermatome leg pain on the seventh day after the operation. The postoperative MRI revealed compression of the $\mathrm{Lt} \mathrm{L} 5$ root and after decompressive laminectomy, the pain improved again. We speculated that the large size of the cage caused the compression of an adjacent nerve root. The reason for the cage migration in 1 patient was not found. In another patient with cage migration, the preoperative bone densitometry (BDM) was -3.5. The severity of the osteoporosis resulted in a vertebral fracture and cage migration.

\section{DISCUSSION}

MISS techniques have been rapidly developing since the early 1990s. Although MISS techniques have not yet been shown to be superior in efficacy to traditional open techniques ${ }^{13)}$, the advantages of MISS techniques, such as reduced postoperative pain, have made these techniques attractive to many spine surgeons. In 2004, Bergey et al. reported on endoscopic lateral transpsoas approach to the lumbar spine ${ }^{15)}$. Before this technique, endoscopic anterior retroperitoneal approach requiring a considerable amount of retraction of the psoas posteriorly was performed. The MIS LLIF, modification of endoscopic lateral transpsoas approach using tubular retractors, has been recently introduced and allows surgeons to gain spinal fusion more simply and easily.

The key difference from the traditional open retroperitoneal approach is based on the muscle-splitting technique through a small skin incision. Direct lateral exposure of the lumbar spine employs a muscle-splitting approach near the mid-axillary line. External and internal abdominal oblique muscles are split in line with their fibers over the target interspace. The transversalis fascia is punctured entering the retroperitoneal space. In the traditional open approach, the psoas muscle is elevated from the lateral surface of the vertebral 
body and retracted posteriorly toward the foramen. A critical difference in MIS LLIF is the continued splitting of the psoas muscle between the imbedded neural structures. For this reason, MIS LUF technique reduces the manipulation of the aorta and vena cava and eliminates the need to violate or retract the peritoneum ${ }^{11}$.

In this study, we retrospectively reviewed 30 patients treated with MIS LLIF. The VAS scores for low back pain and leg pain decreased significantly after surgery. Ralph et al. reported a significant reduction in VAS scores from 7.9 to 2.4 after MIS LLIF in 37 degenerative lumbar pathology patients ${ }^{13)}$. Elowitz et al. also reported a significant reduction from 7.91 to 2.07 for low back pain and from 7.91 to 2.22 for leg pain in 23 lumbar stenosis patients ${ }^{7}$. In cases of scoliosis, Dakwar et al. reported a mean improvement of 5.7 points in 25 patients). This concomitant improvement in VAS scores proves the effectiveness of the MIS LLIF technique as a suitable option for degenerative lumbar disease. The MIS LLIF has advantage of providing wide exposure to the disc space with the ability to place a large cage at target site, which can increase the height of intervertebral disc space more than that of traditional posterior open surgery ${ }^{7,14,17}$. In this study, the height of intervertebral disc space increased with statistical significance as expected. However, the increase in height of the neural foramen was not statistically significant. Although the neural foramen did not increase significantly, leg pain was improved with statistical significance. The increase in disc height is thought to be enough for improvement in leg pain through indirect decompression ${ }^{7,14,17)}$.

Endplate damage was observed in 11 levels (24\%) during the follow-up period. Roders et al. reported the incidence of endplate damage to be as high as $1 \%{ }^{20)}$. Sharma et al. reported 15 cases of endplate damage in 87 operated disc levels ${ }^{22}$. The reason for the relatively high risk of endplate damage is that our series included not only intraoperative endplate damage but also endplate damage seen on the postoperative plain radiographs. The possible causes include violation of the endplate by a shaver, reamer, trial cage, large size of the implant cage, and poor accuracy of the C-arm angle in the operating room. All levels with a damaged endplate finally progressed to cage settling whether it was a standalone cage level or posterior augmentation level. This means that careful surgical technique should be performed in the operation room to prevent endplate damage regardless of the posterior instrumentation. Most levels of the standalone cage levels progressed to cage settling whether there was endplate damage or not. Thus, the standalone cage technique should be avoided unless the candidates have good bone density and stable lumbar spine or slight spine instability without spondylolisthesis. The possible causes for cage settling include osteoporosis, spi- nal instability, and a narrow disc height preoperatively. The relationship between these factors and cage settling needs to be clarified.

Postoperative transient mild weakness of the psoas and sensory changes in the thigh are known to be secondary complications from the pain and swelling during psoas muscle dissection rather than from nerve injury ${ }^{9,10,15,19)}$. It has recently been noted that serious approach-related complications from the transpsoas technique do occur frequently and are likely underreported. In this study, all patients presenting mild weakness of the psoas and sensory changes in the thigh showed no change in the EMG activity upon positioning of the retractor. Moreover, all symptoms improved after few days or few months. For this reason, we speculated that pain and swelling caused the secondary symptoms. Therefore, close inspection of the surgical field for soft tissue dissection, and awareness of the pressure from the retractor upon the iliac crest may reduce the occurrence of transient weakness and sensory changes in the thigh. The overall incidence of transient thigh sensory change (20\%) and psoas weakness (10\%) in our series was similar to the reported incidence in the literature ${ }^{11)}$.

The greatest concern to spinal surgeons is motor deficit, particularly in MISS surgeons performing the MIS LLIF technique. Knight et al. reported 2 cases of permanent motor deficit thought to be caused by injury to the L4 root ${ }^{11)}$ and Housten et al. reported 2 cases of permanent nerve injury ${ }^{9}$. In our series, there was one case of lumbar plexus injury $(3 \%)$ despite the use of intraoperative EMG monitoring. However, this incidence of motor deficit is relatively low compared to an incidence of 0.8 to $3.6 \%$ for instrumented PLFs, 1.0 to $6.1 \%$ for PLIFs, $4.1 \%$ for MIS TLIFs, $6.5 \%$ for endoscopic ALIFs, $1.5 \%$ for open ALIFs, and $0.5 \%$ for MIS decompressions ${ }^{20)}$. The lumbar plexus injury patient underwent the lumbar lateral interbody fusion with tubular dilators operation at the L4-5 disc level, concomitant with the series from Rodger et al. ${ }^{20)}$. Benglis et al. speculated that posterior movement of the retractor blade following the initial dilation noted on the intraoperative lateral fluoroscopy resulted in pressure on the nerve ${ }^{4)}$. By cadaveric analysis, Houten et al..$^{9)}$ revealed the progressive ventral migration of the lumbar plexus with the distal lumbar levels, noting progressive ventral migration from L2 to $L 5$ that places the nerve much closer to the center of the disc at L4-5. For this reason, the risk appears to be principally an issue for the L4-5 disc level. To limit the potential for nerve injury, especially at the L4-5 disc level, particular attention should be given during the dilation steps to look for spontaneous EMG activity from nerve irritation and effective anchoring of the retractor to the vertebral body should be performed to avoid inadvertent posterior migration. Moreover, consideration should be given to using an entry point even 
further anteriorly than used elsewhere in the spine.

One of the major limitations of this study was the short follow-up period. For this reason, there were few levels of bone fusions on the CT scans or plain radiographs in our series. For long-term clinical outcomes, radiological outcomes and complications, regular follow-up is needed. In this study, the VAS scoring system was selected because of its simplicity in scoring patients' pain. However, for a more detailed assessment, other scales like the ODI (Oswestry disability index) or the Short Form-12 (SF-12) questionnaire should be selected.

\section{CONCLUSION}

The MIS LLIF is a safe and effective procedure for degenerative lumbar diseases and is associated with good outcomes and moderately severe complications. Further follow-up is necessary to establish its safety and efficacy.

\section{REFERENCES}

1. AcostaFL, Liu J, Slimack N, Moller D, Fessler R, Koski T: Changes in coronal and sagittal plane alignment following minimally invasive direct lateral interbody fusion for the treatment of degenerative lumbar disease in adults: a radiographic study. J Neurosurg Spine 15(1):92-96, 2011

2. Anand N, Baron EM, Thaiyananthan G, Khalsa K, Goldstein TB: Minimally invasive multilevel percutaneous correction and fusion for adult lumbar degenerative scoliosis: atechnique and feasibility study. J Spinal Disord Tech 21(7):459-467, 2008

3. Benglis DM, Elhammady MS, Levi AD, Vanni S: Minimally invasive anterolateral approaches for the treatment of back pain and adult degenerative deformity. Neurosurgery 63(3 Suppl):191196, 2008

4. Benglis Jr DM, Vanni S, Levi AD: An anatomical study of the lumbosacral plexus as related to the minimally invasive transpsoas approach to the lumbar spine. J Neurosurg Spine 10(2): 139-144, 2009

5. Dakwar E, Cardona RF, Smith DA, Uribe JS: Early outcomes and safety of the minimally invasive, lateral retroperitoneal transpsoas approach for adult degenerative scoliosis. Neurosurg Focus 28(3):E8, 2010

6. Eck JC, Hodges S, Humphreys SC: Minimally invasive lumbar spinal fusion. J Am Acad Orthop Surg 15(6):321-329, 2007

7. Elowitz E, Yanni D, Chwajol M, Starke R, Perin N: Evaluation of indirect decompression of the lumbar spinal canal following minimally invasive lateral transpsoas interbody fusion: radiographic and outcome analysis. Minim Invasive Neurosurg 54(5): 201-206, 2011

8. Gumbs AA, Shah RV, Yue JJ, Sumpio B: The open anterior para- median retroperitoneal approach for spine procedures. Arch Surg 140(4):339-343, 2005

9. Houten JK, Alexandre LC, Nasser R, Wollowick AL: Nerve injury during the transpsoas approach for lumbar fusion. J Neurosurg Spine 15(3):280-284, 2011

10. Karikari IO, Nimjee SM, Hardin CA, Hughes BD, Hodges TR, Mehta AI, et al: Extreme lateral interbody fusion approach for isolated thoracic and thoracolumbar spine diseases: initial clinical experience and early outcomes. J Spinal Disord Tech 24(6): 368-375, 2011

11. Knight RQ, Schwaegler P, Hanscom D, Roh J: Direct lateral lumbar interbody fusion for degenerative conditions: early complication profile. J Spinal Disord Tech 22(1):34-37, 2009

12. Lee YG, Cha JH, Park JS: Clinical outcome of minimally invasive tubular retractor assisted microscopic discectomy in far lateral lumbar disc herniation. Korean J Spine 7(3):155-160, 2010

13. Mobbs RJ, Sivabalan P, Li J: Minimally invasive surgery compared to open spinal fusion for the treatment of degenerative lumbar spine pathologies. J Clin Neurosci 19(6):829-835, 2012

14. Oliveira L, Marchi L, Coutinho E, Pimenta L: A radiographic assessment of the ability of the extreme lateral interbody fusion procedure to indirectly decompress the neural elements. Spine 35(26 Suppl):S331-337, 2010

15. Ozgur BM, Aryan HE, Pimenta L, Taylor WR: Extreme lateral interbody fusion (xlif): A novel surgical technique for anterior lumbar interbody fusion. Spine J 6(4):435-443, 2006

16. Park Y, Ha JW: Comparison of one-level posterior lumbar interbody fusion performed with a minimally invasive approach or a traditional open approach. Spine (Phila Pa 1976) 32(5):537543, 2007

17. Richards JC, Majumdar S, Lindsey DP, Beaupré GS, Yerby SA: The treatment mechanism of an interspinous process implant for lumbar neurogenic intermittent claudication. Spine (Phila Pa 1976) 30(7):744-749, 2005

18. Riedel CJ: Open anterior lumbar interbody fusion. Clin Neurosurg 47:534-540, 2000

19. Rodgers WB, Cox CS, Gerber EJ: Early complications of extreme lateral interbody fusion in the obese. J Spinal Disord Tech 23(6):393-397, 2010

20. Rodgers WB, Gerber EJ, Patterson J: Intraoperative and early postoperative complications in extreme lateral interbody fusion: an analysis of 600 cases. Spine 36(1):26-32, 2011

21. Sasso RC, Kenneth Burkus J, LeHuec JC: Retrograde ejaculation after anterior lumbar interbody fusion: transperitoneal versus retroperitoneal exposure. Spine 28(10):1023-1026, 2003

22. Sharma AK, Kepler CK, Girardi FP, Cammisa FP, Huang RC, Sama AA: Lateral lumbar interbody fusion: clinical and radiographic outcomes at 1 year: apreliminary report. J Spinal Disord Tech 24(4):242-250, 2011

23. Wang MY, Anderson DG, Poelstra KA, Ludwig SC: Minimally invasive posterior fixation. Neurosurgery 63(3):A197-203, 2008

24. Zelko JR, Misko J, Swanstrom L, Pennings J, Kenyon T: Laparoscopic lumbar discectomy. Am J Surg 169(5):496-498, 1995 